\title{
Application de l'immunofluorescence à la détermination de Rhynchosporium secalis sur semences d'orge
}

\author{
B Mille \\ INRA-SRIV (Service de recherches intégrées sur les productions végétales et la protection des plantes), \\ La Verriere, 78320 Le Mesnil-Saint-Denis, France
}

(Reçu le 13 juin 1989; accepté le 13 décembre 1989)

\begin{abstract}
Résumé - Des antisérums ont été obtenus à partir de spores, de mycélium de $R$ secalis et de l'une des toxines émises par le champignon quand il est cultivé sur milieu synthétique. En immunofluorescence indirecte (IF), ils réagissent plus ou moins spécifiquement avec l'agent de la rhynchosporiose de l'orge. La technique a été appliquée à un ensemble de lots de semences et les résultats comparés à ceux obtenus après semis en conditions naturelles : les deux méthodes donnent des résultats voisins. L'utilisation de l'IF pour la détermination des taux de contamination par l'agent pathogène paraît donc envisageable.
\end{abstract}

Rhynchosporium secalis / semence / analyse sanitaire / immunofluorescence

Summary - Use of fluorescence antibody technique to evaluate infection ratio by Rhynchosporium secalis on barley seeds. Antisera was prepared against spores, mycelium of $\mathrm{R}$ secalis and one of the toxins of the fungus produced on synthetic medium. In the indirect fluorescence antibody technique (IF), their dilution limits vary and they react more or less specifically with agents of barley scald disease: occasionally cross-reactions were revealed with other fungus which can be present on barley seeds. This technique was applied to many lots of seeds and results were compared to those obtained after sowing in natural conditions : similar results were obtained between the 2 methods. Use of IF to evaluate infection ratio by fungal pathogen appears possible.

Rhynchosporium secalis / seed / health testing / immunofluorescence

\section{INTRODUCTION}

La rhynchosporiose de l'orge à Rhynchosporium secalis (Oud) Davis est présente dans toutes les zones de culture de l'orge où elle fait régulièrement l'objet d'études. En France, bien que son incidence économique soit très variable suivant les années, elle tend à devenir l'une des maladies les plus préoccupantes de l'orge.

Certaines de ses caractéristiques épidémiologiques sont assez bien connues, notamment l'influence des résidus de cultures laissés sur le champ après les récoltes (Fowler et Owen, 1971; Stedman, 1980; Fitt et al, 1986a et b). D'autres aspects sont plus difficiles à préciser, principalement le rôle des semences en tant que source d'inoculum primaire (Shaner, 1981). Les 2 rai- sons majeures sont la faculté très limitée du champignon à croître sur des milieux artificiels et l'absence de technique permettant son identification sur les grains d'orge. De ce fait, $R$ secalis est le seul agent pathogène transmis par les semences d'orge qui ne soit pas révélé par les analyses sanitaires (Champion, 1982).

Les techniques immunologiques (immunofluorescence, Elisa) sont de plus en plus appliquées pour la détection des micro-organismes phytopathogènes, les bactéries en particulier, mais également les champignons du sol, de la rhizosphère, ou sur la plante elle-même (Hill et Gray, 1967 ; Marchant et Smith, 1968 ; Malajczuck et al, 1975). En revanche, elles sont rarement appliquées en tant que méthodes de mise en évidence des champignons sur les semences 
(OEPP/EPPO, 1987 ; Gleason et al, 1987), mais uniquement, et de façon très récente sur des organes végétaux, tels que des tubercules de pomme de terre (Hingand et al, 1983).

Nous avons essayé d'évaluer si ce type de technique peut convenir à la révélation de l'agent de la rhynchosporiose de l'orge sur les semences et de vérifier, notamment, qu'il est possible d'obtenir des antisérums suffisamment spécifiques du champignon.

\section{MATÉRIEL ET MÉTHODES}

\section{Obtention des immunogènes}

Nous avons utilisé 3 sources d'antigènes pour l'obtention d'immunsérums : les spores, le mycélium et une des toxines émises par le champignon quand il croît sur un milieu artificiel (Auriol et al, 1978).

\section{Les spores}

Elles sont produites par culture du champignon $\left(20^{\circ} \mathrm{C}\right.$, lumière naturelle) sur milieu solide "Lima bean" $(23 \mathrm{~g}$ de farine de Lima bean Difco + $12 \mathrm{~g}$ de gélose par litre d'eau permutée) (Pauvert, communication personnelle) ou "orge" ( $25 \mathrm{~g}$ de farine d'orge variété Jaidor $+12 \mathrm{~g}$ gélose par litre d'eau permutée). La sporulation est abondante sur ces deux milieux entre le $10^{\circ}$ et le $20^{\circ}$ jour et les spores facilement récoltées par lavage des milieux avec de l'eau permutée stérile. La suspension obtenue est filtrée, centrifugée $(10000 \mathrm{~g}, 30 \mathrm{~min})$ et ajustée à $15 \times 10^{6}$ spores $\mathrm{mL}^{-1}$.

\section{Le mycélium}

II est cultivé sur un milieu liquide Czapeck (Rapilly, 1968) enrichi de $20 \mathrm{~g}$ de glucose par litre de milieu (1 litre de milieu par récipient Erlen Meyer de 2 litres) agité par un flux d'air stérile (filtre Millipore Sterivex-GS de porosité $0,22 \mu \mathrm{m}$ ) injecté par un compresseur (500 $\mathrm{cm}^{3} / \mathrm{min}$ par litre de milieu). L'ensemble flacons, filtres et compresseur est disposé dans l'enceinte stérile d'une hotte à flux laminaire. Chaque flacon est ensemencé avec 5 rondelles ( $5 \mathrm{~mm}$ de diamètre) prélevées dans des cultures de $R$ secalis (milieu solide gélosemalt ; $10 \mathrm{~g}$ de cristo malt Difal en poudre par litre d'eau permutée stérile) âgées de 4 semaines. De cette façon, on obtient 10 à $16 \mathrm{~g}$ de mycélium (poids frais) retenu et lavé à l'eau permutée stérile sur filtre de verre fritté (porosité comprise entre 90 et $150 \mu \mathrm{m}$ ). Ce lavage permet d'éliminer le milieu de culture et la presque-totalité des spores présentes au sein du mycélium. Pour l'injection, ce dernier est préalablement mis en suspension (passage au Poter, $3 \mathrm{~g}$ de mycélium/mL d'eau permutée stérile).

\section{La toxine}

Celle utilisée (rhynchosporoside), de nature glycoprotéique, est présente dans les filtrats de culture de
$R$ secalis. Elle a été isolée et purifiée par Mazars (Mazars et al, 1987) pour obtenir une solution titrant $0,5 \mathrm{mg} / \mathrm{mL}$ de protéines solubles dans l'eau (dosage par la méthode Bradford, 1976).

\section{Production des immunsérums}

Quelle que soit la forme antigénique (spore, mycélium ou rhynchosporoside), l'immunisation des lapins (âgés de 4 mois) est réalisée selon un protocole qui comprend 3 injections (L'Haridon, communication personnelle).

Pour la première injection, intradermique multisites, l'antigène est mélangé à l'adjuvant complet de Freund ( $1 \mathrm{~mL}$ de suspension d'antigène $+1,2 \mathrm{~mL}$ d'adjuvant) (Vaitukatis, 1981). La deuxième, 3 semaines plus tard, est intramusculaire dans le rable en 4 sites ( $1 \mathrm{~mL}$ de suspension d'antigène $+1,2 \mathrm{~mL}$ d'adjuvant incomplet de Freund). La troisième, par voie intraveineuse, est réalisée la $6^{\theta}$ semaine $(1 \mathrm{~mL}$ de suspension d'antigène dans l'eau physiologique).

Deux prélèvements sanguins sont effectués, le premier (sérum témoin) avant immunisation, le second 8 jours après la troisième injection. Après formation du caillot, les sérums sont centrifugés et les surnageants conservés au congélateur à $-20^{\circ} \mathrm{C}$.

\section{Technique de caractérisation par immunofluorescence}

La technique d'immunofluorescence indirecte (Faure et al, 1977) a été utilisée. Les lames de verre (Microprint - comportant 10 puits) sont dégraissées à l'éthanol, puis passées dans une flamme 3 ou 4 fois juste avant leur utilisation. Le matériel à analyser (suspension de spores ou de mycélium, broyat végétal) est déposé dans les puits des lames (20 $\mu \mathrm{L}$ /puits) puis séché $\left(40^{\circ} \mathrm{C}, 45 \mathrm{~min}\right)$ et fixé à l'éthanol à $95^{\circ}\left(40^{\circ} \mathrm{C}\right.$, $15 \mathrm{~min})$. Le sérum anti-Rhynchosporium est dilué dans une eau physiologique tamponnée $\left(0,01 \mathrm{M} \mathrm{PO}_{4}^{-} ; \mathrm{pH}\right.$ $7,2)$ additionnée de Tween $20(0,05 \%)$ avant d'être mis en contact avec les dépôts secs $(20 \mu \mathrm{L} /$ puits) pendant $30 \mathrm{~min}$ (enceinte humide à $37^{\circ} \mathrm{C}$ ). Les réactions antigène-anticorps sont révélées au moyen d'un conjugué fluorescent (Biosys) dilué au $1 / 200$ dans la même solution que pour le sérum de lapin (enceinte humide à $37^{\circ} \mathrm{C}$ pendant $30 \mathrm{~min}$ ). Enfin, la préparation est montée dans la glycérine tamponnée $\left(0,1 \mathrm{M} \mathrm{PO}_{4}^{-} ; \mathrm{pH} \mathrm{7,6)}\right.$.

\section{Titres des immunsérums}

Les titres des sérums sont évalués par la technique d'immunofluorescence indirecte précédemment décrite. La suspension de spores est préparée de la même façon que pour l'immunisation des lapins et le mycélium obtenu par broyage de nécroses foliaires dans de l'eau distillée. Des dilutions des antisérums comprises entre $1 / 1$ et $1 / 50000$ sont éprouvées. Les résultats sont comparés à ceux obtenus avec les sérums témoins prélevés avant immunisation. La dilution limite est celle au-delà de laquelle la fluorescence du champignon n'est plus observable. 


\section{Réactions croisées avec d'autres champignons}

Les champignons présents sur les semences d'orge [Alternaria spp, Penicillium spp, Epicoccum nigrum Link, Trichoderma spp, Fusarium roseum var graminearum (Schwabe) SN et $\mathrm{H}$, Helminthosporium gramineum (RAB) et Helminthosporium teres (SACC)] sont isolés et conservés par repiquages sur milieux nutritifs artificiels. La technique d'immunofluorescence est appliquée au mycélium et aux spores de ces champignons afin de déterminer leurs éventuelles affinités avec les différents immunsérums. Une seule dilution est alors utilisée pour chaque type de sérum, correspondant à une concentration double de celle de la dilution limite définie en présence des spores de $R$ secalis, de façon à conduire le travail dans des conditions de concentrations d'anticorps spécifiques similaires.

\section{Application de la technique d'immunofluorescence aux semences d'orge}

Les lots de semences récoltées en 1988 proviennent du domaine de La Verrière et de producteurs privés. Ils présentaient tous des grains tachés. Certaines nécroses sont caractéristiques d'un champignon déterminé (tache semblable à celle observable sur feuille pour $R$ secalis, taches brunes longitudinales pour Fusarium roseum), d'autres non. Suivant les lots, les taches de rhynchosporiose sont soit bien développées, caractéristiques et parfaitement identifiables, soit, dans la majorité des cas, très petites et atypiques, notamment dans les échantillons peu contaminés et leur reconnaissance est alors très difficile ou impossible.

Un échantillon de 100 grains est prélevé dans chaque lot de semences et subit un traitement destiné à éliminer le tégument et les réserves du grain (technique utilisée dans la méthode de détermination des taux de contamination par le charbon nu (Rennie, 1982); ils sont laissés $24 \mathrm{~h}$ dans une solution de soude (100 g de soude par litre d'eau). L'ensemble est ensuite versé sur un tamis à mailles larges $(4 \mathrm{~mm})$ et lavé sous un filet d'eau pour éliminer, notamment, les embryons laissés intacts par le traitement. Seules les glumes sont retenues dans le tamis. Chacune d'elle est broyée individuellement dans $200 \mu \mathrm{L}$ d'eau permutée dans un mortier; $20 \mu \mathrm{L}$ du broyat est déposé dans une alvéole de la lame Microprint.

Certains de ces lots ont ensuite été semés au champ suivant un dispositif de 4 blocs de parcelles $\left(10 \mathrm{~m}^{2}\right)$ randomisées. A la sortie de l'hiver, 500 plantules sont prélevées (stade "4 feuilles") dans chaque parcelle, de façon à déterminer les taux de feuilles cotylédonaires contaminées par $R$ secalis et vérifier la correspondance entre le résultat d'analyse par immuno fluorescence et les taux effectifs de contamination en conditions naturelles.

\section{RÉSULTATS}

\section{Dilutions limites des immunsérums}

\section{En présence de spores de $\boldsymbol{R}$ secalis (tableau I)}

C'est avec l'injection de spores uniquement que nous obtenons la valeur la plus élevée $(1 / 20000)$. Les immunsérums obtenus avec le mycélium seul ou avec les autres formes antigéniques présentent des titres intermédiaires, et l'injection de toxine donne lieu à un résultat faible.

\section{En présence de mycélium de $\boldsymbol{R}$ secalis}

Le classement des immunsérums en fonction de leurs dilutions limites reste sensiblement le même qu'avec. les spores. Les 2 immunsérums obtenus avec des purifications de spores ou de mycélium ont les valeurs les plus fortes $(1 / 20000)$. Ceux obtenus à la fois avec du mycélium et des spores sont moyens et les immunsérums faisant intervenir la toxine sont les plus faibles.

Tableau I. Dilutions limites des immunsérums en présence de spores et de mycélium $R$ secalis. * Sérum prélevé avant immunisation. Dénomination Antigènes injectés
du sérum
2

1

\begin{tabular}{|c|c|c|c|c|c|}
\hline & 1 & 2 & 3 & Spores & Mycélium \\
\hline Témoin * & - & - & - & $1 / 2$ & $1 / 10$ \\
\hline sss & Spores & Spores & Spores & $1 / 2000$ & $1 / 20000$ \\
\hline $\mathrm{mmm}$ & Mycélium & Mycélium & Mycélium & $1 / 500$ & $1 / 10000$ \\
\hline$t t t$ & Toxine & Toxine & Toxine & $1 / 50$ & $1 / 500$ \\
\hline ssm & Spores & Spores & Mycélium & $1 / 250$ & $1 / 2000$ \\
\hline $\mathrm{mms}$ & Mycélium & Mycélium & Spores & $1 / 500$ & $1 / 2000$ \\
\hline $\mathrm{mmt}$ & Mycélium & Mycélium & Toxine & $1 / 250$ & $1 / 500$ \\
\hline
\end{tabular}

Dilution limite du sérum en présence de 


\section{Spécificité des immunsérums}

Quand on confronte les divers immunsérums obtenus à d'autres champignons que $R$ secalis, leurs spécificités s'avèrent différentes (Tableau II).

Les sérums élaborés à partir d'un antigène unique (sss, $\mathrm{mmm}$ et $\mathrm{ttt}$ ) s'avèrent relativement peu spécifiques. Celui correspondant à la toxine (ttt) réagit avec presque tous les champignons, quelle que soit leur forme (spores ou mycélium), et plus particulièrement avec les mycéliums d'Alternaria spp, de Trichoderma viride et de $\mathrm{Hel}$ minthosporium teres. Ceux résultant d'immunisation avec des spores (sss) ou du mycélium $(\mathrm{mmm})$ sont assez équivalents quant à leur niveau de spécificité, mais différents par les champignons avec lesquels ils présentent des réactions.

Le premier sérum (sss) donne lieu à une fluorescence avec les 2 formes de Helminthosporium gramineum, ainsi qu'avec les spores de Penicillium spp et le deuxième (mmm) avec le mycélium de Fusarium graminearum et les spores d'Alternaria spp. Les sérums sss et $\mathrm{mmm}$ présentent également des réactions croisées avec le mycélium de Penicillium spp et Trichoderma viride, et avec les spores d'Epicoccum nigrum, de $H$ teres et de $T$ viride.
Quand, pour la troisième injection de l'immunisation, le mycélium est remplacé par des spores et inversement (sérums ssm et $\mathrm{mms}$ ), la spécificité est améliorée. Comparé au sérum sss, le sérum ssm ne présente plus de réaction avec les spores et mycélium de $H$ gramineum, ainsi qu'avec les spores de Penicillium spp et de Trichoderma viride. De même, par rapport au sérum $\mathrm{mmm}$, mms ne donne plus lieu à un résultat positif en présence de spores de Penicillium spp et de $T$ viride, ainsi qu'avec le mycélium de $F$ graminearum. Toutefois, il se révèle non spécifique vis-à-vis du mycélium de $\mathrm{H}$ gramineum.

Le sérum $\mathrm{mmt}$ semble intermédiaire entre $\mathrm{mmm}$ et $\mathrm{ttt}$. II est beaucoup plus spécifique de $R$ secalis que celui résultant d'injections de toxine uniquement, mais bien moins que $\mathrm{mmm}$. Une fluorescence est notamment observée en présence de $H$ gramineum, de spores de $T$ viride et de $F$ graminearum, et du mycélium d'E nigrum et de $H$ teres.

On peut présenter les immunsérums par ordre de spécificité croissante suivant la nature des antigènes successivement utilisés lors des différents protocoles d'immunisation:

-1) toxine - toxine - toxine (ttt);

-2) mycélium - mycélium - toxine (mmt);

-3) spores - spores - spores (sss);

-4) mycélium - mycélium - mycélium ( $\mathrm{mmm}$ );

Tableau II. Réactions croisées des immunsérums avec divers champignons en immunofluorescence. Sérum témoin prélevé avant immunisation; $s=$ spores; $m=$ mycélium; $t=$ toxine;,,$++++++=$ réaction fluorescente à très fluorescente; $-=$ absence de fluorescence.

\begin{tabular}{|c|c|c|c|c|c|c|c|c|}
\hline \multicolumn{2}{|l|}{ Sérums } & $t t t$ & $m m t$ & sss & $\mathrm{mmm}$ & $\mathrm{mms}$ & ssm & Témoin \\
\hline \multicolumn{2}{|l|}{$\begin{array}{l}\text { Dilutions des } \\
\text { immunsérums }\end{array}$} & $1 / 25$ & $1 / 125$ & $1 / 1000$ & $1 / 250$ & $1 / 250$ & $1 / 125$ & 1 \\
\hline \multirow{2}{*}{$\begin{array}{l}\text { A secalis } \\
\text { (Témoin) }\end{array}$} & $\mathbf{s}$ & +++ & +++ & $++t$ & +++ & +++ & +++ & + \\
\hline & $\mathrm{m}$ & +++ & ++ & + & +++ & ++ & +++ & + \\
\hline \multirow[t]{2}{*}{$H$ teres } & $\mathbf{s}$ & - & - & - & - & - & - & - \\
\hline & $\mathrm{m}$ & +++ & + & \pm & \pm & \pm & + & + \\
\hline \multirow[t]{2}{*}{ H gramineum } & $\mathbf{s}$ & ++ & + & + & - & - & - & - \\
\hline & $m$ & \pm & \pm & + & - & + & - & + \\
\hline \multirow[t]{2}{*}{ F graminearum } & $s$ & - & \pm & - & - & - & - & - \\
\hline & $m$ & + & - & - & \pm & - & - & + \\
\hline \multirow[t]{2}{*}{ Penicillium spp } & $\mathbf{s}$ & \pm & \pm & ++ & \pm & - & - & + \\
\hline & $m$ & \pm & - & - & - & - & - & + \\
\hline \multirow[t]{2}{*}{ E nigrum } & $\mathbf{S}$ & - & - & - & - & - & - & - \\
\hline & $m$ & + & ++ & \pm & \pm & ++ & \pm & + \\
\hline \multirow[t]{2}{*}{ Alternaria spp } & $s$ & + & + & - & \pm & \pm & - & - \\
\hline & $\mathrm{m}$ & +++ & - & - & - & - & - & + \\
\hline \multirow[t]{2}{*}{$T$ viride } & $\mathbf{s}$ & \pm & $+t$ & + & \pm & - & - & + \\
\hline & $\mathrm{m}$ & +++ & ++ & \pm & ++ & ++ & + & + \\
\hline
\end{tabular}


- 5) mycélium - mycélium - spores (mms);

-6) spores - spores - mycélium (ssm).

Le dernier sérum, bien que le plus spécifique de $R$ secalis, présente encore des réactions croisées avec le mycélium de $H$ teres, Trichoderma spp et $E$ nigrum, bien qu'elles soient très atténuées comparées au témoin (mycélium de $R$ secalis).

\section{Analyse immunologique de lots d'orge d'hiver}

Pour l'analyse immunologique des lots de grains, nous avons choisi le sérum le plus spécifique de $R$ secalis (ssm). Quelques rares spores ont été observées (jamais plus de 3 spores par puits), mais la présence du mycélium fluorescent est constatée.

La technique d'immunofluorescence permet donc effectivement de révéler que les taches atypiques sont bien dues à la présence de $R$ secalis, du moins dans le cas de lots récoltés en 1988. Les taux de contamination par la rhynchosporiose sont élevés, entre 5 et $34 \%$ (tableau III).

L'observation des plantules après semis de ces lots en conditions naturelles permet de vérifier la relation entre les résultats de l'analyse en immunofluorescence et le pourcentage de feuilles cotylédonaires atteintes par $R$ secalis. Les valeurs obtenues pour les deux types d'observa-

Tableau III. Relation entre grains estimés contaminés par l'analyse en immunofluorescence et feuilles cotylédonaires nécrosées. (La Verrière, notations en janvier et février 1989). ${ }^{1}$ Résultats de l'analyse par immunofluorescence de 100 grains; 2 Résultats d'observations de 500 feuilles cotylédonaires (exprimés en pourcentage) $;-=$ levée insuffisante ou notation non faite.
Variété

Contaminations
des lots

de semences ${ }^{\prime}$
Feuilles

cotylédonaires contaminées 2

\begin{tabular}{lcc}
\hline Jaidor & 34,0 & 35,8 \\
Alpha & 30,8 & 24,9 \\
Protidor & 27,0 & 30,1 \\
Jaidor & 25,5 & - \\
Metro & 25,8 & 21,8 \\
Panda & 13,5 & 17,3 \\
Fedora & 12,4 & 12,5 \\
Barberousse & 9,8 & 12,2 \\
Antarès & 8,6 & 10,8 \\
Viva lot 1 & 8,1 & - \\
Viva lot 2 & 7,4 & - \\
Plaisant & 6,5 & - \\
Thibaut & 6,2 & 9,2 \\
Nymphe & 6,0 & - \\
Clerix & 5,6 & - \\
\hline
\end{tabular}

tions ne sont pas statistiquement différentes (test de comparaison de couples de moyennes par calcul de l'écart réduit). Les pourcentages sont dans la majorité des cas très proches, l'écart le plus important $(5,9 \%)$ concerne la variété Alpha. Pour les autres lots, la différence ne dépasse pas $4 \%$.

\section{CONCLUSIONS}

Les sérums anti- $R$ secalis sont utilisables pour observer le mycélium et les spores du champignon en immunofluorescence et la méthode d'analyse immunologique constitue un outil adapté à la détermination des taux de contamination des lots de semences par $R$ secalis.

L'amélioration de la spécificité des sérums par immunisations complexes (différentes formes antigéniques injectées au cours d'un même protocole) est importante. Ces protocoles sont très simples ( 3 injections de quantités relativement faibles du champignon) et rapides (entre 7 et 10 semaines). Dans le cas de $R$ secalis, l'injection successive de spores par piqûres intradermiques multisites, suivie à 3 semaines d'une injection intramusculaire de spores puis, à 6 semaines d'une injection intraveineuse de mycélium, donne lieu à l'élimination de la majeure partie des réactions croisées (avec Penicillium spp, Helminthosporium gramineum, Trichoderma viride) obtenues en injectant uniquement des spores ou du mycélium.

Enfin, le sérum obtenu avec la toxine seule (ttt) donne lieu à des résultats très surprenants puisqu'il s'avère être le moins spécifique, alors que la glycoprotéine est totalement liée à la croissance mycélienne de $R$ secalis sur milieu artificiel, sans qu'on puisse émettre d'hypothèse explicative particulière.

\section{DISCUSSION}

Les techniques immunologiques peuvent être envisagées en analyse de routine car elles ne sont pas plus contraignantes, par exemple, qu'une analyse des taux de contamination par le charbon nu (Rennie, 1982), pour laquelle une préparation est également nécessaire, et pour laquelle l'observation se fait embryon par embryon. Les 2 analyses peuvent même être menées de front, la préparation des grains, la séparation des embryons des glumes et l'élimination de l'albumen, pouvant être commune. La méthodologie du test d'analyse définitif peut être améliorée, après une étude biométrique, notamment sur la prise d'échantillon, et la conduite des 
notations. Ce travail peut également être poursuivi en utilisant la technique Elisa pour donner lieu à l'élaboration d'une méthode de routine applicable à l'analyse d'un grand nombre d'échantillons et éviter l'observation au microscope.

Son application en tant qu'analyse sanitaire intéresse les multiplicateurs de semences, notamment en cas de litige avec l'agriculteur (développement important de la rhynchosporiose en culture). II est également possible, dans le cas où les taux de contamination sont importants, d'envisager un traitement des semences dont l'intérêt a déjà été évoqué (Mille, 1987).

Ce travail constitue une première approche des séquences d'immunisations complexes, alors qu'en général les auteurs (Hingand et al, 1983 ; Malajczuk et al, 1975; Gleason et al, 1987) n'utilisent qu'une seule forme antigénique. Toutefois, l'intérêt de cette innovation doit être confirmé : chaque protocole d'immunisation n'a été appliqué qu'à un seul lapin et les résultats devront être validés lors d'un travail comportant des répétitions pour vérifier que les différences de spécificité des sérums sont liés aux séquences d'injections.

\section{REMERCIEMENTS}

L'auteur remercie plus particulièrement Mme $\mathrm{C}$ Vergnet et M. L'Haridon pour leur aide et leurs conseils, ainsi que M. Mazars pour la fourniture de la rhynchosporoside.

\section{RÉFÉRENCES}

Auriol P, Strobel G, Beltran JP, Gray G (1978) Rhynchosporoside, a host selective toxin produced by Rhynchosporium secalis, the causal agent of scald disease of barley. Proc Natl Acad Sci USA 75, $4339-4343$

Bradford MM (1976) A rapid and sensitive method for the quantitation of microgram quantities of protein utilizing the principe of protein-dye binding. Anal Biochem 72, 248-254

Champion R (1982) Les techniques de lutte. Les ennemis à combattre. Colloque sur le traitement des semences de céréales à paille. 27 avril 1982, 30-32

Faure M, Dupouey P, Morelec MJ (1977) La fixation de l'antigène. La pénétration des anticorps marqués dans le matériel antigénique. L'immunofluorescence en milieu liquide et les réactions sur les cellules et les germes vivants. In : Les Techniques de l'immunofluorescence et les réactions immuno-enzymatiques. "Cours de l'Institut Pasteur" Maloine 57-65

Fitt BDL, Creighton NF, Lacey ME, McCartney HA (1986a) Effects of rainfall intensity and duration on dispersal of Rhynchosporium secalis conidia from infected barley leaves. Trans Br Mycol Soc 86, 4, 611-618

Fitt BDL, Walklate PJ, McCartney HA, Bainbridge A, Creighton NF, Hirst JM, Lacey JM, Legg BJ (1986b) $A$ rain tower and wind tunnel for studying the dispersal of plant pathogens by rain and wind. Ann Appl Biol 109, 3, 661-671

Fowler AM, Owen H (1971) Studies on leaf blotch of barley (Rhynchosporium secalis). Trans $\mathrm{Br} \mathrm{Mycol}$ Soc 56, 1, 137-152

Gleason ML, Ghabrial SA, Ferriss RS (1987) Serological detection of Phomopsis longicolla in soybean seeds. Phytopathology 77, 2, 371-375

Hill IR, Gray TRG (1967) Application of the fluorescent antibody technique to an ecological study of bacteria in soil. J Bacteriol 93, 6, 1888-1896

Hingand L, Le Coz S, Kerlan C, Jouan B (1983) Application de l'immunofluorescence à la détection de Phoma exigua var foveata, agent de la gangrène de la pomme de terre. Agronomie 3, 1, 51-56

Malajczuk N, McComb AJ, Parker CA (1975) An immunofluorescence technique for detecting Phytophthora cinnamomi rands. Aust J Bot 23, 289-309

Marchant R, Smith DG (1968) A serological investigation of hyphal growth in Fusarium culmorum. Arch Mikrobiol 63, 85-94

Mazars C, Rossignol M, Auriol P (1987) Purification par HPLC de glycoprotéines phytotoxiques produites par Rhynchosporium secalis (Oud) Davis. Bio-Sciences 6, 3, 63-68

Mille B (1987) La rhynchosporiose de l'orge : possibilité de transmission par la semence? Phytoma 391 , 31-34

OEPP/EPPO (1987) Detection and characterization of pathogenic and symbiotic viruses, bacteria. MLOs and fungi by immunological and biochemical methods. Bull OEPP/EPPO 17, 299-319

Rapilly F (1968) Les techniques de mycologie en pathologie végétale. Ann Epiphyties 19

Rennie WJ (1982) Barley - loose smut (Ustilago nuda (Jens) Rostr). In : ISTA Handbook Health Testing, working sheet, 25

Shaner G (1981) Effect of environment of fungal leaf blights of small grains. Ann Rev Phytopathol 19, 273-296

Stedman OJ (1980) Observations on the production and dispersal of spores and infection by Rhynchosporium secalis. Ann Appl Biol 163-175

Vaitukatis JL (1981) Production of antisera with small doses of immunogen : multiple intradermal injections. Methods Enzymol 73, 46-52 